\title{
The Great Need of a Biomechanical-Based Approach for Surgical Methods of Giant Cell Tumor: A Critical Review
}

\author{
Azadeh Ghouchani $^{1}$ - Gholamreza Rouhi $^{1}$
}

Received: 25 October 2016/Accepted: 27 April 2017/Published online: 22 June 2017

(C) Taiwanese Society of Biomedical Engineering 2017

\begin{abstract}
There are many unanswered questions about giant cell tumor (GCT) treatment and not enough attention is paid to the biomechanics of the current treatment methods. Treatment methods have not changed much, and the best method remains controversial to some degree, due to the lack of adequate clinical and biomechanical investigations. Biomechanical tests, including in vitro mechanical experiments combined with finite element analysis, are very helpful in assessing the efficiency of the surgical methods employed and in determining the optimal method of surgery. Tests can be tailored to meet a patient's needs, while limiting postoperative complications. One of the complications, following tumor surgery, is the frequency of postoperative fractures. In order to prevent postoperative fractures, defect reconstruction is recommended. The reconstruction usually consists of defect infilling with bone cement, and in the case of large defects cement augmentation is employed. Whether cement augmentation is essential and offers enough mechanical strength and what is the best fixation device for cement augmentation are areas of debate. In this article, the biomechanical studies comparing different methods of tumor surgery and cement augmentation, highlighting the areas needing more attention to advance GCT treatment, are critically reviewed. Based on our review, we recommend a biomechanical criterion for the essence of defect reconstruction, which must include patient specific factors, in addition to the tumor geometrical properties.
\end{abstract}

Gholamreza Rouhi

grouhi@uottawa.ca; grouhi@aut.ac.ir

1 Faculty of Biomedical Engineering, Amirkabir University of Technology, 15875-4413, 424 Hafez Ave,

Tehran 1591634311, Iran
Keywords Giant cell tumor - Reconstruction methods . Finite element analysis - Mechanical tests - Biomechanics

\section{Introduction}

Giant cell tumor (GCT) was reported for the first time in early eighteenth century as a benign [1-7], albeit locally aggressive tumor of bone $[1-3,5,6,8]$, which can destroy a large amount of bone rapidly $[4,5,7]$. Its name originates from the large number of multinucleated giant cells presented in the tumor. It is mainly located eccentrically in the metaphyseal-epiphyseal region of long bones [5, 9-12], predominantly around the knee $[1,5,10,12,13]$. This tumor often appears in young adults [1, 3, 9, 13-16].

Even though GCT is extensively investigated, little attention is paid to its treatment and biomechanics. Most of the studies on the treatment outcome of this tumor are clinical and retrospective investigations [3, 13, 17-20]. To the best of our knowledge, there is no study discussing and comparing the treatment methods for the bones affected by GCTs from the biomechanical point of view. GCT general treatment methods have not changed much in the past 30 years, due in some degree to the lack of adequate clinical experiments and biomechanical tests. Therefore, many questions about GCT treatment remain to be answered, and the best treatment method for this kind of tumors remains controversial. Several important aspects of GCT treatment must be addressed in order to achieve better outcomes. Optimal removal and reconstruction methods, biomechanics of different fixation devices used for cement augmentation, introduction of a criterion for filling the defects and using fixation devices are some of the issues requiring further understanding and offering topics for future research. 


\section{GCT Treatment}

The objective of GCT treatment is to remove the tumor and to reconstruct the bone defect in order to regain normal limb function [7]. There are several treatment methods for GCT, these range from chemotherapy, radiotherapy, embolization and cryosurgery, to surgery with the use of a chemical or thermal adjuvant. Among all treatment methods, surgery remains the only effective treatment for GCT, in most cases [6]. Nonetheless, the ideal form of surgery for this tumor, particularly when arising near joints in weight bearing bones, remains controversial. Surgical methods may change from intralesional curettage alone, to a wide resection followed by reconstruction [7]. Reconstruction usually involves cementation, and in the case of large defects, cement augmentation. The surgical treatment of GCT consists of two stages: (1) removal of the tumor, and (2) reconstruction of the defect [21], both of which are discussed below.

\subsection{Tumor Removal}

There are a few methods of tumor removal including curettage alone, curettage followed by adjuvant therapy (for example, cryosurgery), bone resection and amputation [16]. GCT removal has always been a challenging task for the surgeons, because under-curettage can enhance the risk of tumor recurrence, while over-excision can result in postoperative bone fractures [22]. Review of the literature reveals that, among different removal techniques, intralesional curettage is the preferred treatment for most GCTs $[10,23,24]$. In order to completely visualize the tumor cavity as well as obtain adequate curettage $[16,25,26]$ and reduce the stress rising effect $[16,25]$, a large cortical window is first created in the curettage technique. The tumor is removed using a curette, following which filling of the cavity is often necessary. In most cases, the technique of tumor removal and reconstruction is based on the surgeons' experience [27, 28].

\subsection{Defect Reconstruction}

The reconstruction of the defect depends on the technique used for removing the tumor, and it is known that the more drastic the removal, the more difficult the reconstruction will be [21]. After removing the tumor, the cavity can be left unfilled, or it can be filled with cement or bone grafts [17]. There are experimental data demonstrating that the bone defects that are left empty, as well as those filled with a bone substitute, heal gradually [29-31]. Hirn et al. [18] made a retrospective study on the outcome of curettage alone in 146 patients, having benign bone tumors around the knee among which $47 \%$ were diagnosed as GCT. The results showed that some defects were completely filled with new bone, while some were never filled in. It was concluded that the size of the defect is an important factor for development of osteoarthritis or postoperative fracture [18]. In another study [32] on 193 patients, the rate of GCT postoperative fractures was reported to be $4 \%$ after curettage without any adjuvant therapy. In a similar study, Yanagawa et al. [20] reported the outcome of curettage surgery alone in patients with benign bone tumors. Their results indicated incomplete filling of all cavities, and postoperative fractures in 3 patients among 78, even though no weight bearing occurred for about 3 months post-operation [20]. Nonetheless, most of the experts suggested that the cavity after GCT curettage should be filled [33-39]. In GCT surgery, there are several options for filling the cavity and defect reconstruction, including bone grafting with autograft or allograft and a variety of bone substitutes such as polymethylmethacrylate (PMMA) bone cement and hydroxyapatite (HA) [40], with bone cement and allograft as the most frequently used materials [33, 34, 38, 39].

Remodeling of bone grafts occur along the stress lines produced by the applied load on the defected bone according to Wolff's law [41], and when it integrates with neighboring bone a permanent reconstruction is made. The disadvantages of bone grafting however are donor-site complications [41], limited quantity, and its high cost [13, 40, 42, 43]. Filling the cavity with cement provides immediate stability [13, 40, 42, 44], so patients can return to their physical activities earlier. Another advantage of bone cement, as a filler for large defects, is its mechanical properties [10, 19], which are similar to human bone. Cement, like bone, is stronger in compression than tension, and its modulus of elasticity is just slightly greater than that of healthy trabecular bone [45]. Moreover, subchondral stiffness has been indicated to be approximately $98 \%$ of that of the intact contralateral limb, when cement is used for defect reconstruction [44]. With the advantages of PMMA bone cement as the filling material, it is not questionable why this material is widely used in the reconstruction of massive bone defects [19, 45] since 1993 [43]. However, PMMA is not a perfect material for filling bone defects, since there is concern about the radiolucent line at the bone-cement interface. It is not still clear if the radiolucent line is progressive and causes loosening of the cement [43]. Other disadvantages of using PMMA cement are the thermal and chemical necrosis of surrounding bone, due to the high heat generated during PMMA polymerization, and unbound monomer release [46]. 


\section{Treatment Complications}

GCT treatment remains a challenging issue to date because of its frequent post-operative complications. The most common complications following GCT surgery consist of high tumor recurrence rate, pathologic and postoperative fractures, and failed defect reconstruction [2, 42].

Although it has been assumed that gender, age, tumor location, size, or the amount of subchondral bone remaining after tumor removal, may all affect the recurrence rate [13], some studies demonstrate that the only factor influencing tumor recurrence is the adequacy of tumor removal $[13,18,25,26,47]$. Whether or not adjuvants used after curettage can reduce the rate of local recurrence is controversial. Some researchers have concluded that the type of adjuvant used does not have a significant effect on the rate of recurrence [48, 49]. However, other studies have shown lower rates of recurrence following the use of such adjuvants, particularly bone cement [16, 40, 48].

GCT can greatly destroy bone and cause pathologic fractures [22]. Pathologic fracture occurs in approximately $20 \%$ of patients with GCT [50]. The literature also shows pathological fractures in $4-32 \%$ of cases, with an average of $12 \%$ in studies with a large number of patients with GCT [3]. Malawer et al. [51] reported a 5.9\% rate of pathologic fractures among 102 patients with bone GCT treated with cryosurgery, without the use of internal fixation devices for reconstruction. Marcove et al. [52] reported $25 \%$ postoperative fractures among 52 patients with GCT, treated with extended curettage followed by cryosurgery and cement infilling. Thus, the major drawback of the technique of curettage and cementation are the high fracture risk, due to the early loading of the bone and the insufficient fixation of the cement in the cavity [21]. Several methods are developed to reduce or eliminate these drawbacks. Pattijn et al. [53] made a thin metal membrane made of titanium to pack the cavity created after GCT curettage. This membrane was produced preoperatively using computer aided techniques and it attached at the periosteum of the bone around the cavity during surgery [53]. In addition to containing the filling material in the cavity, using this membrane made early normal functioning of patients possible as it restored the strength and stiffness of the bone [53]. Augmenting the cement with internal fixation devices is another method to reduce the risk of fractures. There are biomechanical studies determining if cement augmentation would result in a stronger reconstruction. The stronger and stiffer construct of cement-augmented bone can make earlier walking and rehabilitation after surgery for the patient possible, and decrease the risk of postoperative fractures $[22,44]$.

\section{Biomechanics of Bone Affected by GCT}

Bone continuously remodels itself through a coupled process of bone resorption by osteoclasts, and bone formation via osteoblasts. This process is named the bone remodeling process [54]. Optimal remodeling is responsible for bone strength $[55,56]$. There are several theories which describe the bone remodeling process as a function of mechanical loads, bone microstructure, and biochemical factors [57, 58]. Cancer cells negatively perturb the balance between the activity of osteoclasts and osteoblasts [59], and they may accelerate the bone remodeling process $[60,61]$. This increase of the bone remodeling activity in the presence of cancer cells is due to the higher production rate of some proteins or genes by the defected bone, compared to a normal bone. In 2015, an in vivo study was performed to assess cortical bone remodeling after curettage and cementation [62]. Two cases, where patients introduced with benign bone tumors in the metacarpal bones of the hand, and where their tumors were removed and the remaining defect was filled by injectable calcium-phosphate cement, were studied for 8 years [62]. The results demonstrated complete bone remodeling by the end of the study, as evidenced by the fact that the shape of the metacarpals reconstructed looked close to the contralateral intact side [62].

The existence of a tumor in the bone changes the pattern of load distribution in the affected area. The new pattern of load distribution, due to the tumor, influences the rate of the bone remodeling process. In addition, the change in the biomechanics of the surrounding tissues and structures is more important after tumor surgery. As is mentioned earlier, curettage and reconstruction with bone cement is the most common procedure for GCT treatment [63-65]. Filling the cavity with bone cement can make the load distribution in the affected area uneven and can result in degenerative changes in the neighboring joint [66]. The stress shielding in the areas close to the cement may weaken the cancellous bone over time. As the Young's modulus of cement is higher than that of trabecular bone, when the limb affected by tumor and reconstructed by bone cement is loaded, most of the load is transferred to the stiffer material. This uneven load distribution leads to bone resorption in the surrounding cancellous bone [66]. A long term study, on the effects of cementation after GCT curettage in the weight bearing bones, demonstrated that the defected bone metabolism alters the rate of bone remodeling [67], and consequently it weakens the surrounding trabecular bone as a result of the change in the mechanical load distribution [68]. The adverse effects on the surrounding bone and neighboring joint is even more 


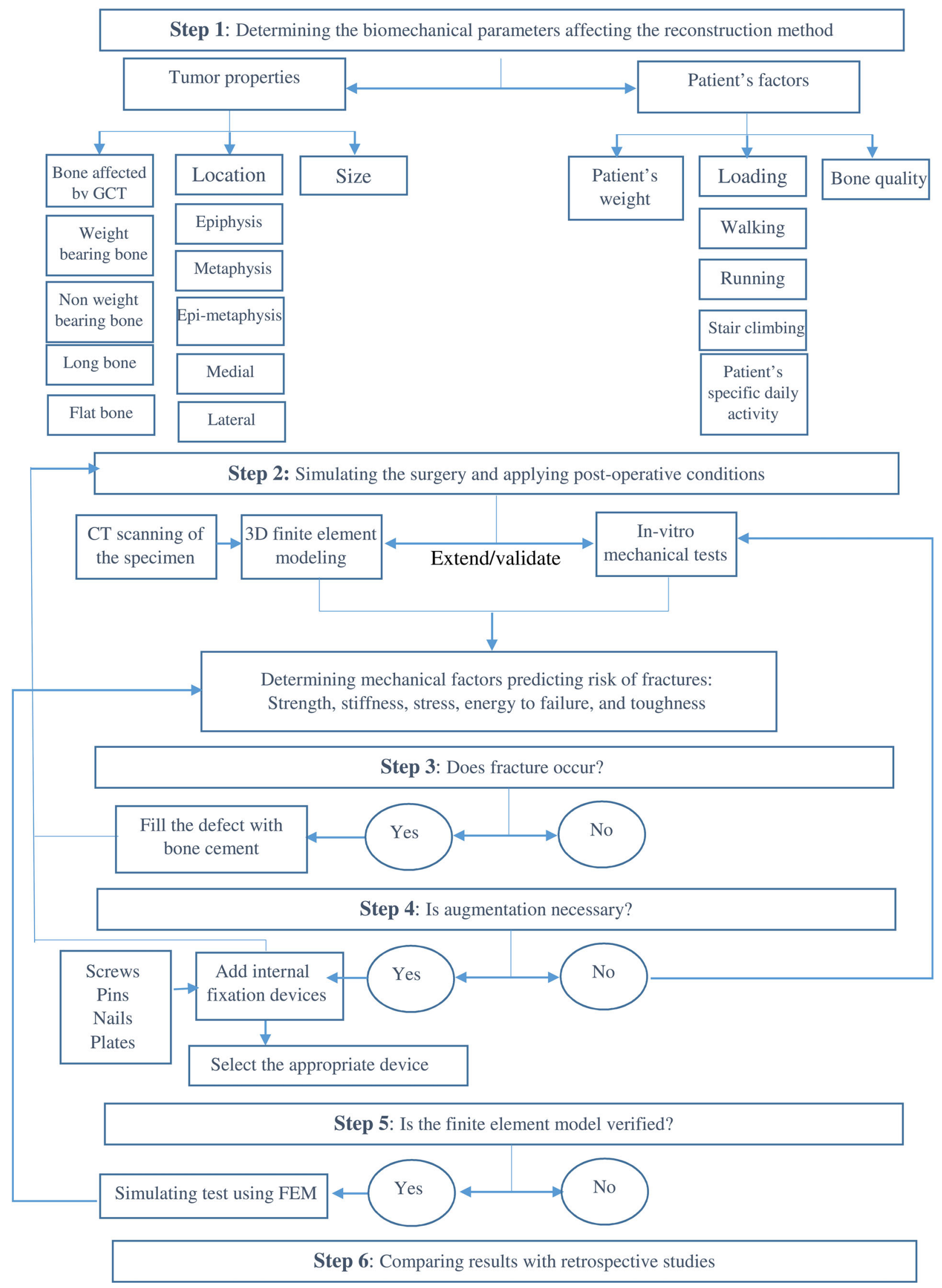


4Fig. 1 Different steps for deriving a biomechanical criterion for the reconstruction method of GCT surgery

critical in the case of extensive curettage and cementation [66]. An in vivo study in dogs was done in order to assess the extent of blood perfusion and remodeling activity following the use of two types of cements [69]. Defects were created in the diaphysis part of the two tibia bones, in which one defect was filled with standard PMMA, and the contralateral defect was filled with a modified bone cement, a cement with a lower polymerization temperature and therefore reduced unbound monomer concentration [69]. The results showed that the standard PMMA bone cement inhibits the vascular response and bone remodeling more than the modified cement [69].

Destruction of the joint surface may occur during aggressive curettage that causes secondary osteoarthritis and degenerative changes in the articular cartilage of the adjacent joint $[64,65]$. Because most GCTs occur around the knee, reduction in the thickness of subchondral bone, due to extensive curettage, can destroy the articular cartilage, and results in secondary degenerative changes in the knee. Suzuki et al. [64] analyzed the effect of residual thickness of subchondral bone on the local recurrence and secondary osteoarthritis in patients with GCT around the knee. Their results indicated that secondary osteoarthritis may occur if the residual thickness of subchondral bone is less than $5 \mathrm{~mm}$. Re-operations due to tumor recurrence, or postoperative fractures, are also significant factors associated with osteoarthritis progression [64]. Szalay et al. [67] obtained similar results by introducing the subchondral thickness as a diagnostic tool for assessing the risk of joint damage. In addition, they compared the effects of bone grafting and cementation on the degenerative changes in weight bearing joints following GCT curettage [67]. Their results indicated less degenerative changes in patients with cementation compared with the patients with bone grafting after 24 months [67]. Nonetheless, the rate of degeneration were similar in both groups after 50 months [67]. Another study investigated the effect of bone grafting on the inhibition of secondary osteoarthritis [65]. Seventy six patients with GCT around the knee treated with curettage and cementation, with or without bone grafting in the subchondral bone, were analyzed [65]. Their results demonstrated larger degenerative changes in patients treated with bone cement reconstruction alone, compared to those for whom bone grafting was also used [65]. In addition, the risk of developing degenerative arthritis was high in patients with the residual thickness of the subchondral bone less than $10 \mathrm{~mm}$ after curettage, and thus the use of bone grafts in such cases was recommended [65].

There are concerns about the strength of the reconstructed bone against fractures, and the appropriate reconstruction method, in which the risk of postoperative fractures is minimized, is still a matter of debate [44]. In order to reduce the risk of fractures when a large cavity is present, particularly in load bearing bones such as the femur and tibia, the use of internal fixation devices, including screws, nails, pins, and locking plates is strongly suggested. Cement augmentation with internal fixation devices provides immediate stability, as well as structural support for large defects [9]. Nevertheless, there is still a controversy over the use of fixation devices, thus repairing large defects remains a challenging issue in orthopedic surgery [51]. Biomechanical approaches, including in vitro mechanical tests combined with finite element analyses (FEA) as shown in Fig. 1, can be very helpful in deriving a criterion for determining the proper reconstruction method after GCT curettage. This criterion must involve defect geometry and patients' specific factors, so that the effects of most mechanical parameters influencing postoperative fractures can be assessed. This criterion can then identify whether cement infilling or cement augmentation is necessary, in addition to determining the proper fixation device for this augmentation.

\subsection{Mechanical In Vitro Tests}

In order to predict and compare the success of different reconstruction methods, and so that the surgeon can choose the most suitable method for the patient, the strength and stability of the reconstructed bone should be assessed. Invitro biomechanical tests are very useful for evaluating the mechanical stability, strength, and stiffness of a particular reconstruction method prior to the surgery.

Some specialists [33, 37, 38, 45] use cement augmentation with Steinmann pins, and apply biomechanical tests in order to investigate the benefits of Steinmann pins for reinforcing the bone cement. Asavamongkolkul et al. [33] performed in vitro tests on eight pairs of distal femurs, some filled with PMMA alone, and some with Steinmann pin reinforcement under uni-axial compression load. They compared the strength of these two reconstructions. The results indicated no significant difference in failure load, stiffness, yield point, and the total energy absorbed to failure, between specimens repaired with cement alone and the cement augmented group with Steinmann pins [33].

Murray et al. [35] carried out in vitro mechanical tests to find out if the use of Steinmann pins in cement augmentation results in biomechanical advantages for the contained lateral femoral condyle defects. Their results also demonstrated that stiffness, energy to failure, and maximum load to failure, between the specimens reconstructed with cement alone and cement augmented with pins, were similar [35]. Although some studies [33, 35] do not indicate any biomechanical advantages with Steinmann pin 

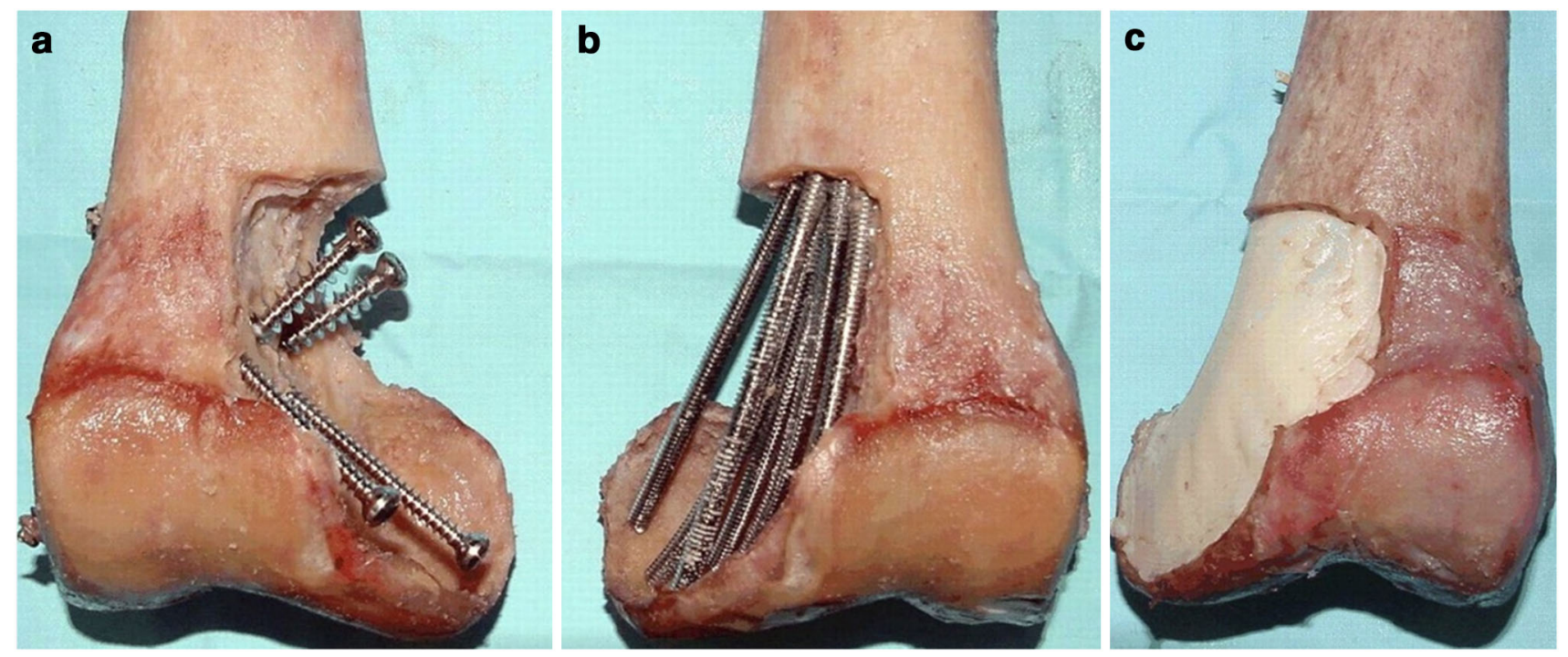

Fig. 2 Configuration of the crossed-screw (a) and the intramedullary Steinmann-pins before cementation (b) and a defect reconstructed with cement only (c) [44]

reinforcement in cement, compared with PMMA reconstruction alone, some biomechanical studies suggested different results. Bini et al. [70] reported successful use of Steinmann pins in augmenting the cement for large defects in 38 patients treated with curettage and cementation. Randall et al. [45] augmented the cement with three intramedullary Steinmann pins in noncontained defects in the proximal part of the tibia, and compared them biomechanically with the contralateral tibiae reconstructed with cement alone. It is worth noting that noncontained defects have no remaining bone cortex, while contained defects refer to those having an intact cortical rim [71]. The specimens were subjected to 2000 compressive cycles, then loaded to failure. The results showed that specimens augmented with pins could better withstand the loads [45]. In contrast to Randall's results [45], the biomechanical study of Weiner et al. [39], on pins for cement augmentation of contained defects in proximal tibia indicated no statistical differences between the biomechanical properties of specimens treated with cement alone and the cement augmented with pins. Weiner et al. [39] applied a load with a constant rate of displacement on each tibia, and they calculated failure load and stiffness from the resulting force-displacement curve. The results demonstrated that cement, augmented with Steinmann pin constructs, were approximately equivalent to the contralateral tibiae reconstructed with cement alone [39]. However, it should be noted that Weiner et al. [39] tested the specimens under one cycle of compressive loading, while Randall et al. [45] tested 2000 cycles of compressive loading. In addition, the type of defects in these two studies was different: being contained in Weiner and co-workers' study [39], while non-contained in Randall and co-workers' study [45].
Toy et al. [44] compared three methods of defect reconstruction in the distal part of the femur: cement alone, cement augmented with intramedullary pins, and cement augmented with screws as indicated in Fig. 2. They applied a compressive force over the medial condyle of each specimen, and the force began at zero load and displacement and increased at a rate of $10 \mathrm{~N} / \mathrm{s}$ until a load of $475 \mathrm{~N}$ was reached [44]. The specimens were then cycled in a sinusoidal pattern between $50 \mathrm{~N}$ and $900 \mathrm{~N}$ for 2000 cycles at $1 \mathrm{~Hz}$, and those specimens that survived the cyclic force were then loaded under a controlled displacement rate until failure [44]. From the force-displacement curves, failure load and stiffness were then calculated [44]. This loading condition was also used in similar studies [38, 45], where they compared cement augmented constructs with specimens treated with cement alone. In this in vitro study [44], cement augmentation with crossed screws provided a stronger reconstruction.

In addition to pins and screw, plates are also commonly used in the reconstruction of the defects created following GCT curettage [38, 72, 73]. Uglialoro et al. [38] created defects in the distal lateral condyle of femurs and filled them with PMMA bone cement, then fixed them with locking plates, Steinmann pins, or crossed screws in order to compare the strength of these devices. In axial load to failure, they observed no difference between specimens reconstructed with cement augmented with Steinmann pins and crossed screws. However, under axial load-to-failure, the locking plate showed greater strength in axial load to failure, compared to Steinmann pins and crossed screws. Ruskin et al. [73] performed an in vitro study on 30 tibias to investigate the stiffness of Steinmann pin with locking plates for augmentation of cement in reconstruction of 
defects remaining after GCT removal. They tested the specimens in a material testing machine for uniaxial compressive load of $400 \mathrm{~N}$ with the rate of $5 \mathrm{~N} / \mathrm{s}$, and they applied a cyclic sinusoidal load between 200 and $1200 \mathrm{~N}$ at $3 \mathrm{~Hz}$ in order to determine the fatigue properties of the constructs. The constructs augmented with locking plates showed greater stiffness and two specimens reconstructed with Steinmann pins failed under cyclic loading [73].

Ahmadi et al. [72] evaluated the biomechanics of retrograde nail, lateral locking plate, and lateral non-locking periarticular plate for stabilization of a simulated metastatic lesion in the distal end of the femur. They created a spherical tumor-like defect that involved half of the circumference of the bone in the lateral metaphyseal region of 15 synthetic femurs and filled the defects with bone cement fixed with one of the three fixation devices mentioned. Mechanical tests for axial, sagittal, coronal, torsional stiffness, and torsional strength were applied. Three-point bending stiffness tests in the sagittal plane and off-set torsional stiffness tests indicated that the nails have better performance than the plates. Therefore, the authors recommended retrograde nails as the optimal device for tumor-like defects in the distal femur, because damage to the surrounding soft tissue was reduced [72].

\subsection{Finite Element Analyses}

Finite element analysis (FEA) and finite element modelling in the field of orthopedic biomechanics has gained wide application. Rouhi et al. [58, 74] applied FEA to investigate the remodeling process in spongy bone, using a semimechanistic bone remodeling theory. They created a cubic model of spongy bone and simulated the remodeling process under microdamage caused by overload. Isotropic and linearly elastic materials were assumed for bone elements. Forces were applied on all six faces of this cube via six plates. The results demonstrated a change in the trabecular orientation and thickness by the change in the direction and amount of load applied [58]. FEA is also applied in several studies to improve the design of implants [75] or increase the efficiency of surgeries by comparing the outcome of different implants [76-84], design optimal orthopedic surgeries [85], and to validate a biomaterial or technique for a better outcome in bone surgery [27]. FEA is able to mimic loading conditions and extend data analysis far beyond what can be practical in a laboratory [75]; hence, it reduces the time and the cost of in vivo and in vitro experimentations. Sophisticated computer models of bone and joints, based on computed tomography (CT) images, can predict the strength of a bone with a simulated defect or reconstructed bone defect as what is seen in GCT surgery [86]. These models provide a deeper understanding of the surgery. A review of literature in this area highlights the dearth of information and research available. However, there are few studies, in which computer simulation techniques for GCT surgeries are used [21, 22, 53].

Finite element simulations can also help to assess stress distribution in the defected areas and the surrounding bone, and to compute stiffness, strength, and energy to failure of each construct. Chanasakulniyom et al. [27] employed FEA into analyze the maximum von Mises stress on different biomaterials used to fill the cavity after GCT removal at distal femurs. The distal femoral bone models were divided into ten parts (Fig. 3), and then different parts of the bones were replaced with PMMA or HA. The stresses were calculated under walking and stair climbing loading conditions, and the results indicated higher von Mises stress of HA in comparison to PMMA. Thus, the authors suggested HA as a better filling material than PMMA in bone replacement, because it has a higher load capacity [27]. The aforementioned study [27] was extended by Aroonjarattham et al. [87] in 2015 to also assess the effect of filling direction and adding an internal fixation device (a locking plate) for HA augmentation. They created a 3D model of the entire femur, proximal tibia, and knee joint including the meniscus and surrounding ligaments using CT images. They assigned different materials properties for cortical and cancellous bone, and all the materials involved in the model were assumed to be linear elastic and isotropic. The distal end of the femur was divided into four parts and the parts were replaced with PMMA or HA from medial to lateral and vice versa (Fig. 4), and the models were loaded from the femur head under walking and stair climbing conditions [87]. Their results demonstrated lower von Mises stress when PMMA

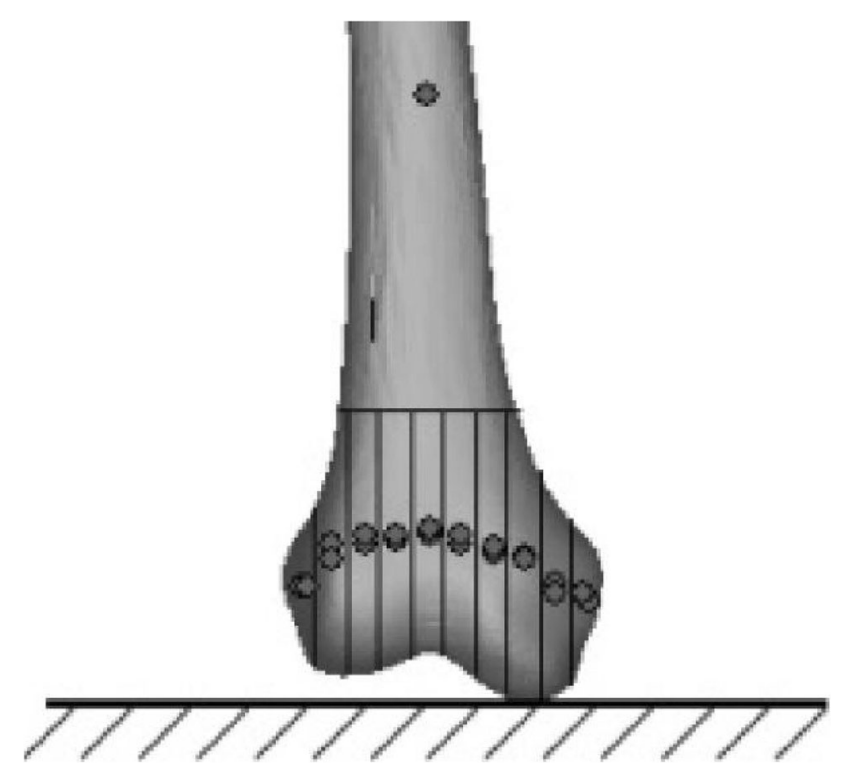

Fig. 3 The distal end of the femur is divided into ten parts, and each part is then replaced with PMMA or HA [27] 
Fig. 4 Models of distal femur demonstrating the replacement area (black) filled with biomaterials from the lateral to medial side (cases $A-D$ ), and the medial to lateral side (cases E-H) [87]

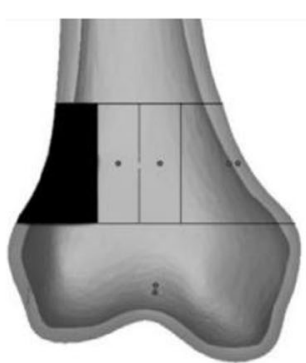

Case A

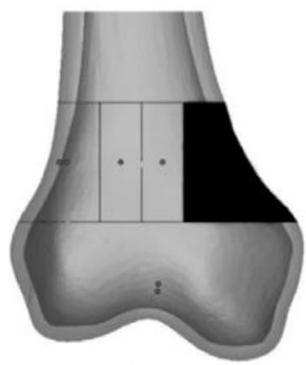

Case E

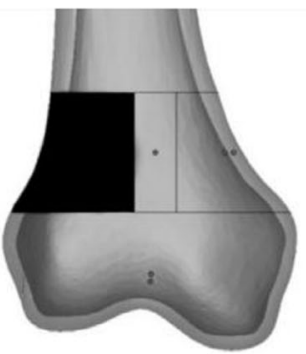

Case B

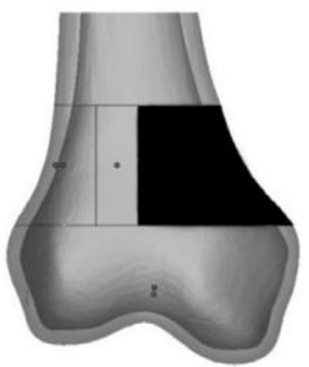

Case F

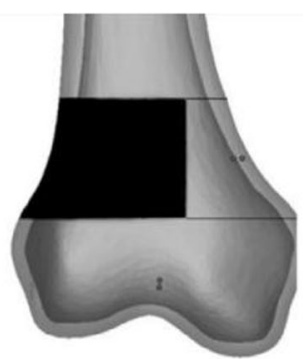

Case C

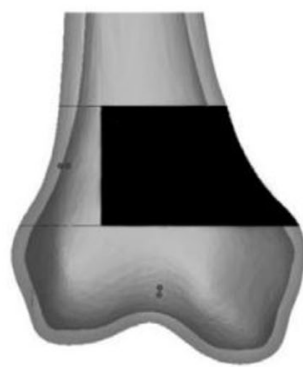

Case $\mathrm{G}$

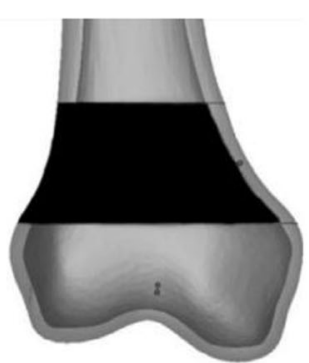

Case D

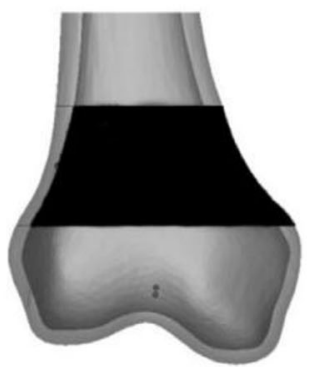

Case $\mathrm{H}$ was replaced from the lateral to medial side, but no significant change in the maximum von Mises stress was detected with regard to the filling direction when the parts were replaced with HA [87].

To improve the accuracy of GCT curettage, Li et al. [22] employed FEA for selecting the best surgical procedure and then performed that procedure in an in vitro condition. They simulated femoral GCT curettage on a patientspecific computer 3D model using CT images. Then they converted the defective femur to a plastic model to be used as a guidance model during surgery. They created a heterogeneous model of bone reconstructed with cement based on the greyscale values of CT images, and three reconstruction methods were modeled to select the best method of surgery. The methods included no filler, filled with cement, and filled with cement and implants [22]. The GCT tumor was then replaced with PMMA, and the implants were added. The boundary conditions were modeled so that the proximal end of the specimens or models was fixed, and loads were applied to the distal part (the condyles). Each medial condyle of the construct was subjected to a compression load (Fig. 5) with a translation rate of $10 \mathrm{~mm} /$ minute, to calculate the stress in cement and implant, as well as the stiffness of each construct. Cement augmented with locking plate, the stiffest construct, was selected as the proper defect reconstruction method [22].

In order to determine the critical size of bone GCT in the distal femur region for cement augmentation, a voxel-based finite element modeling approach, based on the block diagram shown in Fig. 1, was recently proposed [88]. Quantitative CT (QCT) of several cadaveric femoral allografts of both intact and reconstructed with cement, similar to GCT surgery, were taken. Using QCT images, the Hounsfield units (HUs) were converted to bone mineral densities (Fig. 6). Based on the bone mineral densities, Young's modulus of each element was calculated to create a heterogonous model of the bone. Different sizes and locations of GCT surgery were simulated in the distal femur region of the model, and a load similar to in vitro tests was applied in the FE analysis. In order to validate the FEM, the specimens were then tested under a compressive load to failure to calculate the strength of the bones. Based on the reduction in the reconstructed bone strength compared with intact bone and considering patient daily activities, the risk of postoperative fracture can be determined, and the critical size of GCT for which cement augmentation is necessary can be estimated [88, 89].

In another study, QCT based structural rigidity analysis combined with FE analysis was used to predict postoperative fractures of GCT surgery [90]. Mosleh et al. [90] created an isotropic and heterogeneous model of bone reconstructed with bone cement, using QCT images for FE analysis, and they calculated the fracture load by applying uniaxial compression load on the medial condyle of the bone. In addition, they performed the structural rigidity analysis for the weakest cross section of the bone to determine the fracture load [90]. Their results showed that both of these two different approaches correlate well with the loads calculated by the in vitro mechanical tests they performed.

Owing to the high heat generated during PMMA polymerization, bone cement is assumed to eliminate remaining 
Fig. 5 CT image of the GCT in the distal femur and its computer model, anterior view (a). Computer model with an implant, posterior view (b). FE model of the defect filled with bone cement (c) and augmented with a locking plate, posterior view (d). Cement-plus-lockingplate model loaded by an implant, the proximal end is fixed and the load is applied on the medial condyle (e) [22]

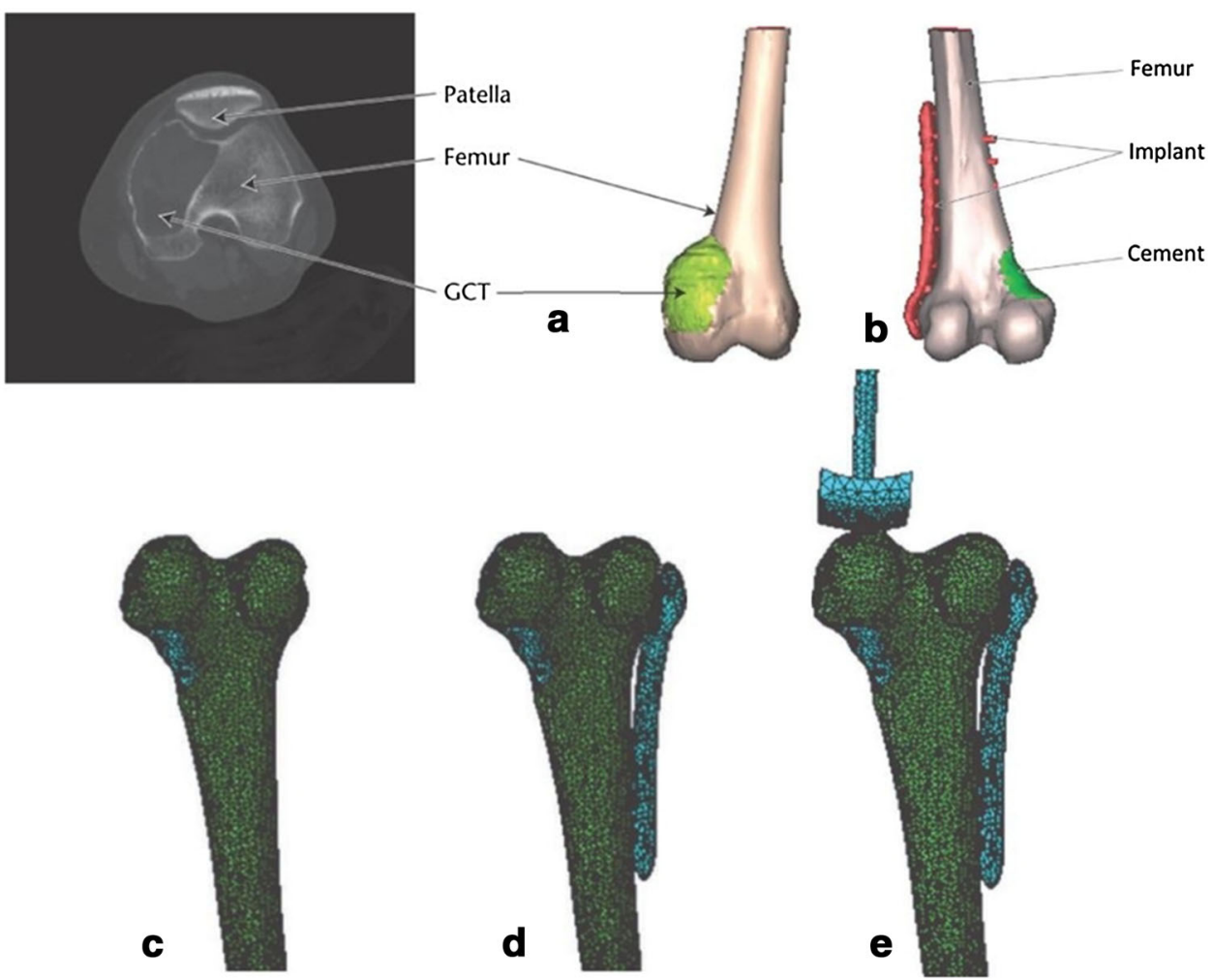

tumor cells [23], resulting in a decreased rate of local recurrence [10, 43, 91]. However, this high heat may cause thermal necrosis of surrounding bone and cartilage. Radev et al. [5] applied FEA to find out the extent of possible thermal necrosis of tissues around PMMA placed into a metaphyseal defect in the proximal tibia. They made a finite element model of a bone with a defect filled with bone cement to simulate the situation after GCT surgery as illustrated in Fig. 7. The model includes articular cartilage, subchondral bone, a prismatic defect filled with cement, and the surrounding cancellous bone. They modeled different thicknesses of remaining cancellous and subchondral bone between the cement region and the articular cartilage. Their results indicated that the risk of thermal necrosis of subchondral bone is serious if there was $<3 \mathrm{~mm}$ of cancellous bone between the PMMA and subchondral bone layer. In addition, in order to prevent thermal necrosis of the adjacent articular cartilage, there should be at least $2 \mathrm{~mm}$ of uniform subchondral bone above the PMMA implant [5]. These results suggest bone cement as a safe filling material after GCT curettage, as long as the continuity of the articular cartilage is preserved and the remaining thickness of subchondral bone is adequate $[5,43]$. It should be noted that the depth of the necrotic region depends on the size of the cement region. When the defect filled with bone cement is large, as expected, the risk of damage to the surrounding healthy tissues substantially increases [92]. Recently, a new composite PMMA was produced in which a phase change material, paraffin, was mixed with cement powder [93]. This new composite compared with common bone cement has a lower exothermic temperature when polymerized in the body [93], and likely has less destructive impact on neighboring bone.

\section{Discussion and Conclusions}

This article reviews complications associated with the surgical treatment of GCTs. One of the major complications is the high percentage of postoperative fracture, thus care must be taken in the selection of surgical methods. Invitro mechanical tests and FEA are crucial and helpful tools in determining an appropriate surgical method with minimum risk of postoperative fracture. Thus, we reviewed biomechanical studies by comparing the different GCT post-operative outcomes, and we introduced a generic biomechanical approach to determine the appropriate GCT surgery method. Moreover, we discussed the biomechanics of bone affected by GCT, in the presence of the tumor and after replacing it with PMMA bone cement.

GCT surgery usually consists of tumor removal and defect reconstruction. On the basis of clinical observations, several studies suggest that the reconstruction of the defect after tumor removal is not necessary [18, 20, 29, 32], except for large defects [36, 42], while others 

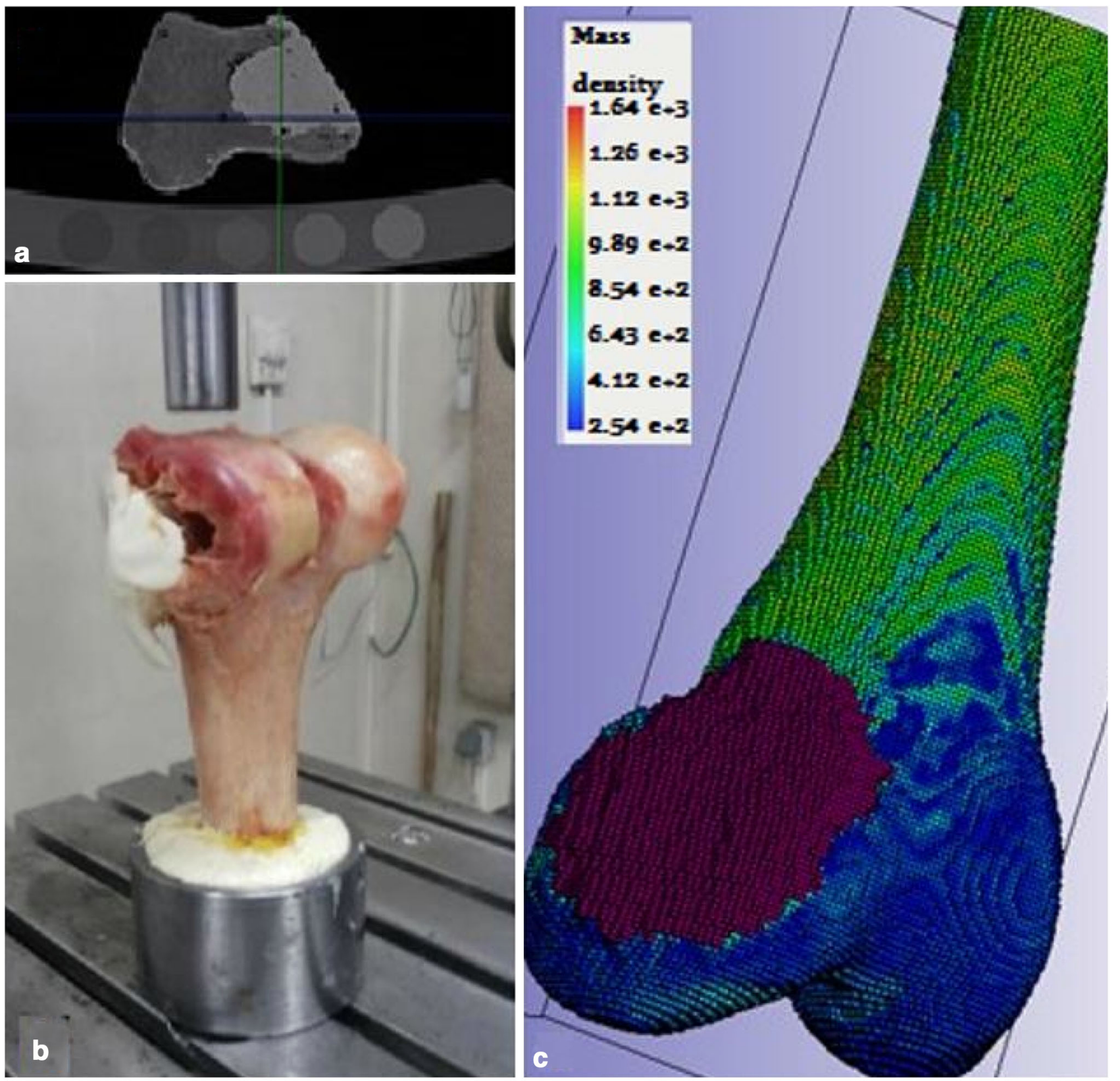

Fig. 6 Transverse view of a QCT scan of bone-cement region and the calibration phantom (a), bone reconstructed with bone cement under compression load (b), and a voxel-based FE model of reconstructed bone showing the heterogamous nature of bone (c) [88]

recommended filling the defect after tumor removal regardless of the size of the tumor $[25,33,35,37,38]$. Hence, no consensus has been reached on the necessity of defect-reconstruction in the current literature related to GCT. This inconsistency can be a motivation to move towards finding a criterion which can determine whether or not the defects created after tumor removal should be filled, and if so, which kind of materials/devices should be employed. Such a criterion should quantitatively describe the critical defect size that needs reconstruction. In the current literature, this critical size is qualitatively described as "large defects" [36, 42].

Among several surgical methods, the review of the literature done in this research reveals that curettage and packing with PMMA bone cement has gained the widest acceptance for the treatment of bone GCTs [10, 19, 42, 45]. Nonetheless, and despite its wide acceptance, it should be noted that this technique may result in degenerative osteoarthritis, thermal necrosis of the surrounding tissues, and even fractures. High stiffness of PMMA (in 


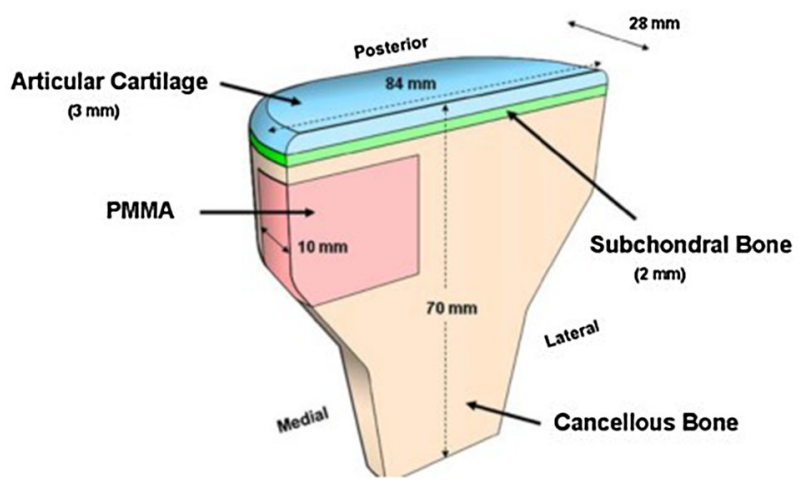

Fig. 7 A schematic FE model of proximal tibia with a simulated defect filled with PMMA bone cement. Due to sagittal plane symmetry, only half of the model is shown [5]

comparison to the surrounding bone) results in an increased strain on the surrounding area which in turn enhances the risk of fractures [66]. Incomplete, and undercurettage, can lead to recurrence, and a delay in the diagnosis of recurrence may cause destruction and secondary osteoarthritic changes in the neighboring joint [94]. Hence, future research should be aimed at identifying reliable clinical factors for predicting the risk of local recurrence, determining the factors influencing the degenerative changes in the adjacent joint, and improving the properties of the filling materials, or making new materials in order to overcome the shortcomings of the currently used filling agents. High fracture risk, as a result of early loading of the bone after surgery and the insufficient fixation of the bone cement in the cavity, is another concern $[22,25]$. Thus, when there is either extensive loss of cortical bone or the defects are large, internal fixation devices should accompany the reconstruction [36-38, 42]. Long-term follow-up studies (with large numbers of patients with GCT) strongly suggest utilization of these devices [51], as it considerably reduces postoperative fractures. Currently, devices such as locking plates, Steinmann pins, screws, and intramedullary nails are usually used [22, 35, 37, 38, 44]. Although there is almost consensus about using fixation devices in the case of large defects, the choice of device for optimal fixation for defects, especially those around the knee, remains unclear.

In order to determine the optimal device and reconstruction method for lesions occurring near the knee (the most pertinent site of GCT occurrence), experimental tests and finite element analyses should be performed $[22,33,35,37,39,44,45]$. In these biomechanical studies, the specimens reconstructed with different methods are usually subjected to a compression load, in cyclic [44, 45] or quasi-static manner [33, 38], and the stiffest and strongest construct is chosen as the optimal treatment method $[35,38,44,72]$. However, in these tests, the effects of tumor geometry, patient's bone quality, weight and daily activities were disregarded in the selection of the internal fixation device and/or reconstruction method. Hence, the choice of the appropriate treatment, which can fit the patient-specific requirements, remains a substantially challenging task for surgeons. Finite element modelling can be a powerful tool in determining the best treatment method for a specific patient prior to the surgery. Different surgery methods can be simulated on the patient's bone using CT images and loading conditions similar to the patient's daily activities. Thus, the most suitable treatment method can be found according to patient-specific factors, tumor size, and location, using finite element investigations. Needless to emphasize that in order to have a reliable and useful finite element model for clinical practice, the model must be validated first. For instance, in vitro tests can be performed on human cadavers and/or synthetic bone specimens. The combination of experimental mechanical tests with finite element modelling can shed light on the currently used reconstruction techniques from a biomechanics point of view. It also makes it possible to derive a logical criterion, which can be employed to come up with a safer surgical approach compared to the current methods of surgery. Treatment strategies might then be modified according to the size, and the location (epiphyseal, metaepiphyseal, or soft tissue involvement) of the defect, as well as patient-specific factors including bone quality, daily physical activities, and the patient's weight. We hope that through using the approach suggested in this article, a universal agreement can be reached on the reconstruction method of giant cell tumors.

Acknowledgements The authors would like to thank Orthopedic Research Center of Mashhad University of Medical Sciences, especially Dr. MH Ebrahimzadeh for his constructive comments during the course of preparation of this review article.

\section{Compliance with ethical standards}

Conflict of interest The authors declare that they have no competing interests.

\section{References}

1. Anwar Ul Haque, A. M. (2008). Giant cell tumor of bone: A neoplasm or a reactive condition? International Journal of Clinical and Experimental Pathology, 1(6), 489.

2. Chakarun, C. J., Forrester, D. M., Gottsegen, C. J., Patel, D. B., White, E. A., \& Matcuk, G. R., Jr. (2013). Giant cell tumor of bone: Review, mimics, and new developments in treatment. $R a$ diographics, 33(1), 197-211.

3. Jeys, L., Suneja, R., Chami, G., Grimer, R., Carter, S., \& Tillman, R. (2006). Impending fractures in giant cell tumours of the distal femur: Incidence and outcome. International Orthopaedics, $30(2), 135-138$. 
4. McDonald, D. J. S. F. M. R. D. D. (1986). Giant-cell tumor of bone. J Bone Joint Surg [Am], 68(2), 235-242.

5. Radev, B. R., Kase, J. A., Askew, M. J., \& Weiner, S. D. (2009). Potential for thermal damage to articular cartilage by PMMA reconstruction of a bone cavity following tumor excision: A finite element study. Journal of Biomechanics, 42(8), 1120-1126.

6. Singh, S., Singh, M., Mak, I., \& Ghert, M. (2013). Expressional analysis of GFP-tagged cells in an in vivo mouse model of giant cell tumor of bone. The Open Orthopaedics Journal, 7, 109-113.

7. Wei, Y., Ek, E. T., Yu, L., \& Yin, G. (2008). Oncological and functional results following operation for giant cell tumour of bone. Journal of Nanjing Medical University, 22(2), 107-110.

8. Malek, F., Krueger, P., Hatmi, Z., Malayeri, A., Faezipour, H., \& O'Donnell, R. (2006). Local control of long bone giant cell tumour using curettage, burring and bone grafting without adjuvant therapy. International Orthopaedics, 30(6), 495-498.

9. Bickels, J., Meller, I., \& Malawer, M. (2001). The biology and role of cryosurgery in the treatment of bone tumors. In M. M. Malawer \& P. H. Sugarbaker (Eds.), Musculoskeletal cancer surgery (pp. 135-145). Dordrecht: Springer.

10. Klenke, F. M., Wenger, D. E., Inwards, C. Y., Rose, P. S., \& Sim, F. H. (2011). Giant cell tumor of bone: Risk factors for recurrence. Clinical Orthopaedics and Related Research, 469(2), 591-599.

11. Cowan, R. W., \& Singh, G. (2013). Giant cell tumor of bone: A basic science perspective. Bone, 52(1), 238-246.

12. Sherwani, R. K., Zaheer, S., Sabir, A. B., \& Goel, S. (2008). Giant cell tumor along with secondary aneurysmal bone cyst of scapula: A rare presentation. International journal of shoulder surgery, 2(3), 59-61.

13. Jamshidi, K., Sami, S., Modarres-Nejad, H., \& Jahansoz, A. (2008). Local recurrence in giant cell tumor of bone: Comparative study of two methods of surgical approach. Journal of Research in Medical Sciences, 13(5), 223-229.

14. Balke, M., Neumann, A., Szuhai, K., Agelopoulos, K., August, C., Gosheger, G., et al. (2011). A short-term in vivo model for giant cell tumor of bone. BMC Cancer, 11(1), 241.

15. Wülling, M., Engels, C., Jesse, N., Werner, M., Delling, G., \& Kaiser, E. (2001). The nature of giant cell tumor of bone. Journal of Cancer Research and Clinical Oncology, 127(8), 467-474.

16. Khalil, E. S., Younis, A., Aziz, S., \& El Shahawy, M. (2004). Surgical management for giant cell tumor of bones. Journal of the Egyptian National Cancer Institute, 16(3), 145-152.

17. Errani, C., Ruggieri, P., Asenzio, M. A. N., Toscano, A., Colangeli, S., Rimondi, E., et al. (2010). Giant cell tumor of the extremity: a review of 349 cases from a single institution. Cancer Treatment Reviews, 36(1), 1-7.

18. Hirn, M., de Silva, U., Sidharthan, S., Grimer, R. J., Abudu, A., Tillman, R. M., et al. (2009). Bone defects following curettage do not necessarily need augmentation: A retrospective study of 146 patients. Acta Orthopaedica, 80(1), 4-8.

19. Tunn, P., \& Schlag, P. (2002). Giant cell tumor of bone. An evaluation of 87 patients. Zeitschrift fur Orthopadie und ihre Grenzgebiete, 141(6), 690-698.

20. Yanagawa, T., Watanabe, H., Shinozaki, T., \& Takagishi, K. (2009). Curettage of benign bone tumors without grafts gives sufficient bone strength: A case-series of 78 patients. Acta Orthopaedica, 80(1), 9-13.

21. Pattijn, V., Gelaude, F., Vander, J., \& Van, R. (2005). Medical image-based preformed titanium membranes for bone reconstruction. Medical Imaging Systems Technology, Methods in General Anatomy, 5, 43-78.

22. Li, J., Wodajo, F., Theiss, M., Kew, M., \& Jarmas, A. (2013). Computer simulation techniques in giant cell tumor curettage and defect reconstruction. Computing in Science \& Engineering, 15(2), 21-26.
23. Pan, K., \& Chan, W. (2010). Curettage and cementation in giant cell tumour of the distal tibia using polypropylene mesh for containment: A case report. Malaysian Orthopaedic Journal, 4(2), 51-53.

24. Yang, T., Zheng, X.-F., Li, M., Lin, X., \& Yin, Q.-S. (2013). Stimulation of osteogenic differentiation in stromal cells of giant cell tumour of bone by zoledronic acid. Asian Pacific Journal of Cancer Prevention, 14(9), 5379-5383.

25. Ayerza, M. A., Aponte-Tinao, L. A., Farfalli, G. L., Restrepo, C. A. L., \& Muscolo, D. L. (2009). Joint preservation after extensive curettage of knee giant cell tumors. Clinical Orthopaedics and Related Research, 467(11), 2845-2851.

26. Puri, A., \& Agarwal, M. (2007). Treatment of giant cell tumor of bone: Current concepts. Indian journal of orthopaedics, 41(2), $101-108$

27. Chanasakulniyom, M., Aroonjarattham, K., Aroonjarattham, P., Saktaveekulkit, N., Sitthiseripratip, K., \& Mahaisavariya, B. (2011). The effect of distal femur after replace with biomaterials: Finite element analysis. Paper presented at the The second TSME International conference on mechanical engineering Krabi, 19-21 October.

28. Benevenia, J., Rivero, S. M., Moore, J., Ippolito, J. A., Siegerman, D. A., Beebe, K. S., \& Patterson, F. R. (2016). Supplemental Bone Grafting in Giant Cell Tumor of the Extremity Reduces Nononcologic Complications. Clinical Orthopaedics and Related Research ${ }^{\circledR}, 1-8$.

29. Lemperle, S. M., Calhoun, C. J., Curran, R. W., \& Holmes, R. E. (1998). Bony healing of large cranial and mandibular defects protected from soft-tissue interposition: A comparative study of spontaneous bone regeneration, osteoconduction, and cancellous autografting in dogs. Plastic and Reconstructive Surgery, 101(3), 660-672.

30. Medige, J., Mindell, E., \& Doolittle, T. (1982). Remodeling of large, persistent bone defects. Clinical Orthopaedics and Related Research, 169, 275-290.

31. Virolainen, P., Heikkilä, J., Yli-Urpo, A., Vuorio, E., \& Aro, H. (1997). Histomorphometric and molecular biologic comparison of bioactive glass granules and autogenous bone grafts in augmentation of bone defect healing. Journal of Biomedical Materials Research, 35(1), 9-17.

32. Prosser, G., Baloch, K., Tillman, R., Carter, S., \& Grimer, R. (2005). Does curettage without adjuvant therapy provide low recurrence rates in giant-cell tumors of bone? Clinical Orthopaedics and Related Research, 435, 211-218.

33. Asavamongkolkul, A., Pongkunakorn, A., \& Harnroongroj, T. (2003). Stability of subchondral bone defect reconstruction at distal femur: comparison between polymethylmethacrylate alone and steinmann pin reinforcement of polymethylmethacrylate. Journal of the Medical Association of Thailand, 86(7), 626-633.

34. Ayerza, M. A., Aponte-Tinao, L. A., Muscolo, D. L., \& Abalo, E. D. (2006). Morsellized and structural cortical allograft reconstruction after intralesional curettage of a distal femoral giant-cell tumor. Orthopedics, 29(8), 679-682.

35. Murray, P. J., Damron, T. A., Green, J. K., Morgan, H. D., \& Werner, F. W. (2004). Contained femoral defects: Biomechanical analysis of pin augmentation in cement. Clinical Orthopaedics and Related Research, 420, 251-256.

36. Ofluoglu, O. (2008). Aggressive treatment of giant cell tumour with multiple local adjuvants. Acta Orthopaedica Belgica, 74(6), 831.

37. Toy, P. C., Arthur, S., Brown, D., \& Heck, R. K. (2007). Reconstruction of noncontained proximal tibial defects with divergent screws and cement. Clinical Orthopaedics and Related Research, 459, 167-173.

38. Uglialoro, A. D., Maceroli, M., Beebe, K. S., Benevenia, J., \& Patterson, F. R. (2009). Distal femur defects reconstructed with 
polymethylmethacrylate and internal fixation devices: A biomechanical study. Orthopedics, 32(8), 561-567.

39. Weiner, M., Damron, T. A., Patterson, F. R., Werner, F. W., \& Mann, K. A. (2004). Biomechanical study of pins in cementing of contained proximal tibia defect. Clinical Orthopaedics and Related Research, 419, 232-237.

40. Lin, P. P., \& Frink, S. J. (2004). Intralesional treatment of bone tumors. Operative Techniques in Orthopaedics, 14(4), 251-258.

41. Calori, G., Mazza, E., Colombo, M., \& Ripamonti, C. (2011). The use of bone-graft substitutes in large bone defects: Any specific needs? Injury, 42, S56-S63.

42. Fraquet, N., Faizon, G., Rosset, P., Phillipeau, J.-M., Waast, D., \& Gouin, F. (2009). Long bones giant cells tumors: treatment by curretage and cavity filling cementation. Orthopaedics \& Traumatology: Surgery \& Research, 95(6), 402-406.

43. Wada, T., Kaya, M., Nagoya, S., Kawaguchi, S., Isu, K., Yamashita, T., et al. (2002). Complications associated with bone cementing for the treatment of giant cell tumors of bone. Journal of orthopaedic science, 7(2), 194-198.

44. Toy, P. C., France, J., Randall, R. L., Neel, M. D., Shorr, R. I., \& Heck, R. K. (2006). Reconstruction of noncontained distal femoral defects with polymethylmethacrylate and crossed-screw augmentation: A biomechanical study. The Journal of Bone and Joint Surgery, 88(1), 171-178.

45. Randall, R., Weenig, K., West, J., Johnston, J., \& Bachus, K. (2002). Durability and strength of Steinmann pin augmentation in cemented tibial defects. Clinical Orthopaedics and Related Research, 397, 306-314.

46. Stańczyk, M., \& Van Rietbergen, B. (2004). Thermal analysis of bone cement polymerisation at the cement-bone interface. Journal of Biomechanics, 37(12), 1803-1810.

47. Blackley, H., Wunder, J., Davis, A., White, L., Kandel, R., \& Bell, R. (1999). Treatment of giant-cell tumors of long bones with curettage and bone-grafting*. The Journal of Bone and Joint Surgery, 81(6), 811-820.

48. Algawahmed, H., Turcotte, R., Farrokhyar, F., \& Ghert, M. (2010). High-speed burring with and without the use of surgical adjuvants in the intralesional management of giant cell tumor of bone: a systematic review and meta-analysis. Sarcoma, 2010, $1-5$.

49. Turcotte, R. E., Wunder, J. S., Isler, M. H., Bell, R. S., Schachar, N., Masri, B. A., et al. (2002). Giant cell tumor of long bone: a Canadian Sarcoma Group study. Clinical Orthopaedics and Related Research, 397, 248-258.

50. Van der Heijden, L., Dijkstra, P. S., Campanacci, D. A., Gibbons, C. M. H., \& van de Sande, M. A. (2013). Giant cell tumor with pathologic fracture: Should we curette or resect? Clinical Orthopaedics and Related Research, 471(3), 820-829.

51. Malawer, M. M., Bickels, J., Meller, I., Buch, R. G., Henshaw, R. M., \& Kollender, Y. (1999). Cryosurgery in the treatment of giant cell tumor: A long term followup study. Clinical Orthopaedics and Related Research, 359, 176-188.

52. Marcove, R. C., Weis, L. D., Vaghaiwalla, M. R., \& Pearson, R. (1978). Cryosurgery in the treatment of giant cell tumors of bone. Clinical Orthopaedics and Related Research, 134, 275-289.

53. Pattijn, V., Samson, I., Sloten, J., Van Audekercke, R., Swaelens, B., \& De Buck, V. (2002). Medical image based, preformed titanium membranes for bone reconstructions: Design study and first clinical evaluation. Proceedings of the Institution of Mechanical Engineers. Part H, Journal of Engineering in Medicine, 216(1), 13-21.

54. Rouhi, G., Epstein, M., Sudak, L., \& Herzog, W. (2006). Free surface density and microdamage in the bone remodeling equation: Theoretical considerations. International Journal of Engineering Science, 44(7), 456-469.
55. Rouhi, G. (2011). A tri-phasic mixture model of bone resorption: Theoretical investigations. Journal of the Mechanical Behavior of Biomedical Materials, 4(8), 1947-1954.

56. Rouhi, G., Epstein, M., Sudak, L., \& Herzog, W. (2007). Modeling bone resorption using mixture theory with chemical reactions. Journal of Mechanics of Materials and Structures, 2(6), 1141-1155.

57. Rouhi, G., Herzog, W., Sudak, L., Firoozbakhsh, K., \& Epstein, M. (2004). Free surface density instead of volume fraction in the bone remodeling equation: Theoretical considerations. Forma, 19(3), 165-182.

58. Rouhi, G., Vahdati, A., Li, X., \& Sudak, L. (2015). A three dimensional computer model to simulate spongy bone remodelling under overload using a semi-mechanistic bone remodelling theory. Journal of Mechanics in Medicine and Biology, 15(04), 1550061.

59. Krishnan, V., Vogler, E. A., Sosnoski, D. M., \& Mastro, A. M. (2014). In vitro mimics of bone remodeling and the vicious cycle of cancer in bone. Journal of Cellular Physiology, 229(4), 453-462.

60. Juan Juan, Y., Pollock, C. B., \& Kelly, K. (2005). Mechanisms of cancer metastasis to the bone. Cell Research, 15(1), 57-62.

61. Wu, P.-F., Tang, J.-Y., \& Li, K.-H. (2015). RANK pathway in giant cell tumor of bone: Pathogenesis and therapeutic aspects. Tumor Biology, 36(2), 495-501.

62. Ichihara, S., Vaiss, L., Acciaro, A., Facca, S., \& Liverneaux, P. (2015). External bone remodeling after injectable calcium-phosphate cement in benign bone tumor: Two cases in the hand. Orthopaedics \& Traumatology: Surgery \& Research, 101(8), 983-986.

63. Pan, K., \& Chan, W. (2010). Curettage and cementation in giant cell tumour of the distal tibia using polypropylene mesh for containment: A case report. Malaysian Orthopaedic Journal, 4(4), 51-53.

64. Suzuki, Y., Nishida, Y., Yamada, Y., Tsukushi, S., Sugiura, H., Nakashima, H., et al. (2007). Re-operation results in osteoarthritic change of knee joints in patients with giant cell tumor of bone. The Knee, 14(5), 369-374.

65. Xu, H., Niu, X., Zhang, Q., Hao, L., Ding, Y., \& Li, Y. (2013). Subchondral bone grafting reduces degenerative change of knee joint in patients of giant cell tumor of bone. Chinese Medical Journal (England), 126, 3053-3056.

66. Gunzburg, R., Szpalski, M., \& Aebi, M. (2007). Vertebral tumors: Philadelphia: Lippincott Williams \& Wilkins.

67. Szalay, K., Antal, I., Kiss, J., \& Szendroi, M. (2006). Comparison of the degenerative changes in weight-bearing joints following cementing or grafting techniques in giant cell tumour patients: Medium-term results. International Orthopaedics, 30(6), 505-509.

68. Kundu, Z. S., Gupta, V., Sangwan, S. S., \& Rana, P. (2013). Curettage of benign bone tumors and tumor like lesions: A retrospective analysis. Indian Journal of Orthopaedics, 47(3), 295.

69. Stümp, J., Nimb, L., \& Jørgen, S. J. (1995). Blood perfusion and remodelling activity in canine tibial diaphysis after filling with a new bone cement compared to bone wax and poly (methyl methacrylate) cement. Biomaterials, 16(11), 845-848.

70. Bini, S., Gill, K., \& Johnston, J. O. (1995). Giant cell tumor of bone. Curettage and cement reconstruction. Clinical Orthopaedics and Related Research, 321, 245-250.

71. Qiu, Y. Y., Yan, C. H., Chiu, K. Y., \& Ng, F. Y. (2011). Review article: Bone defect classifications in revision total knee arthroplasty. Journal of Orthopaedic Surgery, 19(2), 238.

72. Ahmadi, S., Shah, S., Wunder, J. S., Schemitsch, E. H., Ferguson, P. C., \& Zdero, R. (2013). The biomechanics of three different fracture fixation implants for distal femur repair in the presence of a tumor-like defect. Proceedings of the Institution of Mechanical Engineers. Part H, Journal of Engineering in Medicine, 227(1), 78-86. 
73. Ruskin, J., Caravaggi, P., Beebe, K. S., Corgan, S., Chen, L., Yoon, R. S., et al. (2016). Steinmann pin augmentation versus locking plate constructs. Journal of Orthopaedics and Traumatology, 17, 1-6.

74. Rouhi, G., Vahdati, A., Li, X., \& Sudak, L. J. (2015). An investigation into the effects of osteocytes density and mechanosensitivity on trabecular bone loss in aging and osteoporotic individuals. Biomedical Engineering Letters, 5(4), 302-310.

75. Zdero, R., \& Bougherara, H. (2010). Orthopaedic biomechanics: A practical approach to combining mechanical testing and finite element analysis. In D. Moratal (Ed.), Finite element analysis. Vienna: Intech Education and Publishing.

76. Samsami, S., Saberi, S., Bagheri, N., \& Rouhi, G. (2016). Interfragmentary motion assessment for three different fixation techniques of femoral neck fractures in young adults. Bio-Medical Materials and Engineering, 27(4), 389-404.

77. Nourisa, J., \& Rouhi, G. (2016). Biomechanical evaluation of intramedullary nail and bone plate for the fixation of distal metaphyseal fractures. Journal of the Mechanical Behavior of Biomedical Materials, 56, 34-44.

78. Kamal, Z., \& Rouhi, G. (2016). A parametric investigation of the effects of cervical disc prostheses with upward and downward nuclei on spine biomechanics. Journal of Mechanics in Medicine and Biology, 16(2), 1650092 (1650091-1650014).

79. Akbarian, D., Rouhi, G., Mashhadi, M. M., \& Herzog, W. (2015). Biomechanics of cervical spine following implantation of a semiconstrained artificial disc with upward center of rotation: A finite element investigation. Journal of Mechanics in Medicine and Biology, 15(04), 1550063.

80. Haase, K., \& Rouhi, G. (2013). Prediction of stress shielding around an orthopedic screw: Using stress and strain energy density as mechanical stimuli. Computers in Biology and Medicine, 43(11), 1748-1757.

81. Nourisa, J., Baseri, A., Sudak, L., \& Rouhi, G. (2015). The effects of bone screw configurations on the interfragmentary movement in a long bone fixed by a limited contact locking compression plate. Journal of Biomedical Science and Engineering, 8(9), 590.

82. Samsami, S., Saberi, S., Sadighi, S., \& Rouhi, G. (2015). Comparison of three fixation methods for femoral neck fracture in young adults: Experimental and numerical investigations. Journal of Medical and Biological Engineering, 35(5), 566-579.

83. Rouhi, G., Tahani, M., Haghighi, B., \& Herzog, W. (2015). Prediction of stress shielding around orthopedic screws: Timedependent bone remodeling analysis using finite element approach. Journal of Medical and Biological Engineering, 35(4), $545-554$.
84. Haase, K., \& Rouhi, G. (2010). A discussion on plating factors that affect stress shielding using finite element analysis. Journal of Biomechanical Science and Engineering, 5(2), 129-141.

85. Murphy, S., Kijewski, P., Simon, S., Chandler, H., Griffin, P., Reilly, D., et al. (1986). Computer-aided simulation, analysis, and design in orthopedic surgery. The Orthopedic Clinics of North America, 17(4), 637-649.

86. Snyder, B. D., Hipp, J. A., \& Nazarian, A. (2009). Non-invasive prediction of fracture risk due to metastatic skeletal defects. Clinical Cancer Research, 15(24), 7676-7683.

87. Aroonjarattham, P., Aroonjarattham, K., \& Chanasakulniyom, M. (2015). Biomechanical effect of filled biomaterials on distal Thai femur by finite element analysis. Kasetsart Journal (Natural Science), 49, 263-276.

88. Ghouchani, A., Ebrahimzadeh, M. H., \& Rouhi, G. (2016). Voxel-based finite element model of a reconstructed bone: Simulating a bone tumor surgery. Journal of Bioengineering \& Biomedical Science, 6(5), 47. doi:10.4172/2155-9538.C1.014.

89. Ghouchani, A., Ebrahimzadeh, M. H., \& Rouhi, G. (2016). Predicting the risk of post-operative fracture of giant cell tumor using fully voxelised finite element models. In 24th annual meeting of the Iranian orthopaedic association, Tehran, Iran, 26-30 September 2016 (pp. 76-77).

90. Mosleh, H., Rouhi, G., Ghouchani, A., Nourisa, J., \& Bagheri, N. (2017). Prediction of the fracture risk of reconstructed bone with cement using QCT based structural rigidity and finite element analysis. Paper presented at the Orthopaedic Research Society Annual Meeting San Diego, California.

91. Becker, W., Dohle, J., Bernd, L., Braun, A., Cserhati, M., Enderle, A., et al. (2008). Local recurrence of giant cell tumor of bone after intralesional treatment with and without adjuvant therapy. The Journal of Bone and Joint Surgery, 90(5), 1060-1067.

92. Nelson, D., Barker, M., \& Hamlin, B. (1997). Thermal effects of acrylic cementation at bone tumour sites. International Journal of Hyperthermia, 13(3), 287-306.

93. Lv, Y., Li, A., Zhou, F., Pan, X., Liang, F., Qu, X., et al. (2015). A novel composite PMMA-based bone cement with reduced potential for thermal necrosis. ACS Applied Materials \& Interfaces, 7(21), 11280-11285.

94. McGough, R. L., Rutledge, J., Lewis, V. O., Lin, P. P., \& Yasko, A. W. (2005). Impact severity of local recurrence in giant cell tumor of bone. Clinical Orthopaedics and Related Research, 438, $116-122$. 